\title{
Neural patterns in linguistic cortices discriminate the content of verbal working memory
}

Benjamin Kowialiewski ${ }^{1,2}$, Laurens Van Calster ${ }^{1,3}$, Lucie Attout ${ }^{1}$, Christophe Phillips ${ }^{1,2}$, Steve Majerus ${ }^{1,2}$

${ }^{1}$ University of Liège, Liège, Belgium

${ }^{2}$ Fund for Scientific Research - F.R.S.-FNRS, Brussels, Belgium

${ }^{3}$ University of Geneva, Geneva, Switzerland

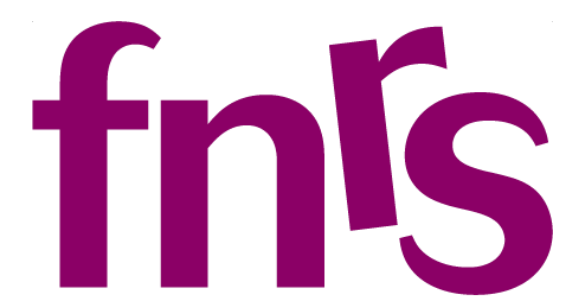

LA LIBERTÉ DE CHERCHER
Psychologie \& Neuroscience Cognitives

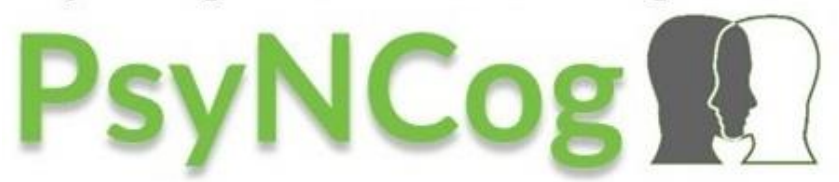

Psychology \& Neuroscience of Cognition
- LIÈGE université 


\section{Introduction}

Verbal working memory: temporary maintenance of verbal information

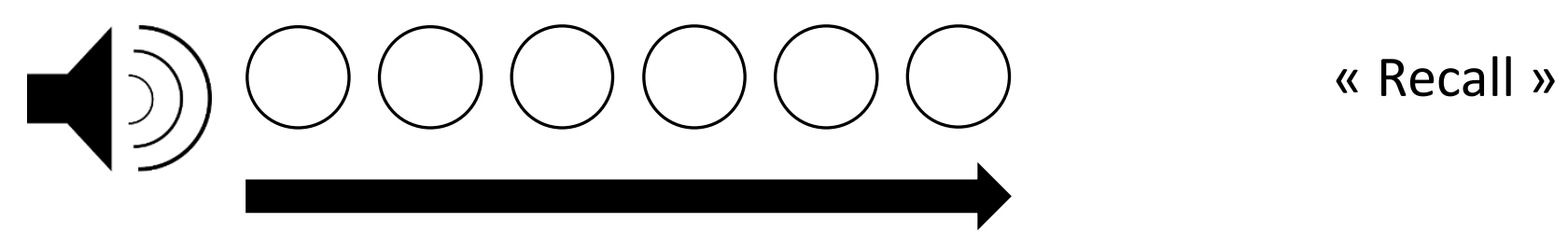




\section{Introduction}

Psycholinguistic effects:

Increased recall performance for verbal items associated to richer or more robust linguistic representations. 


\section{Introduction}

Lexicality effect

Words muscle - phase - ruche - doute - main

Nonwords mique - zisse - jousse - raibe - fanle 


\section{Introduction}

\section{Working memory}

Where and how do they occur?

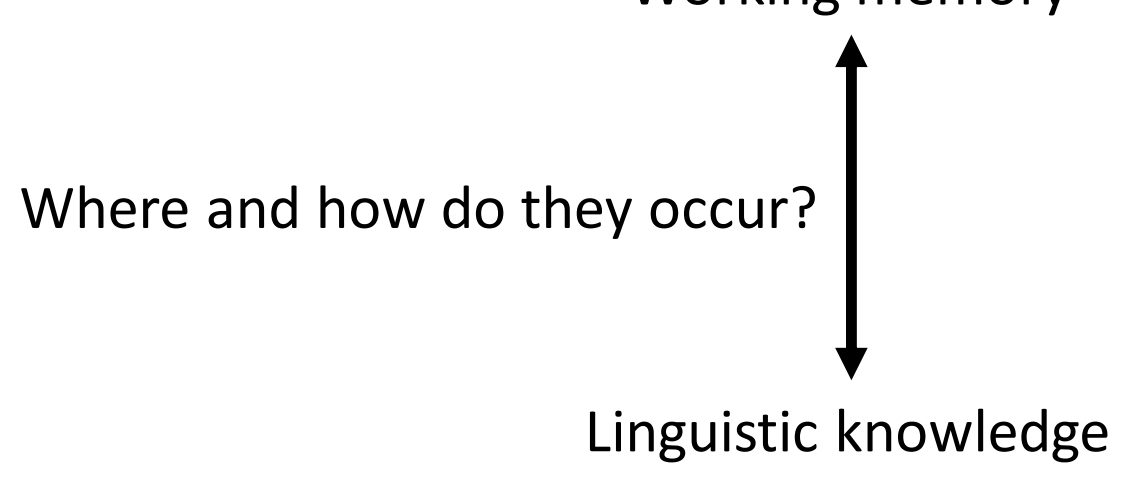




\section{Introduction}

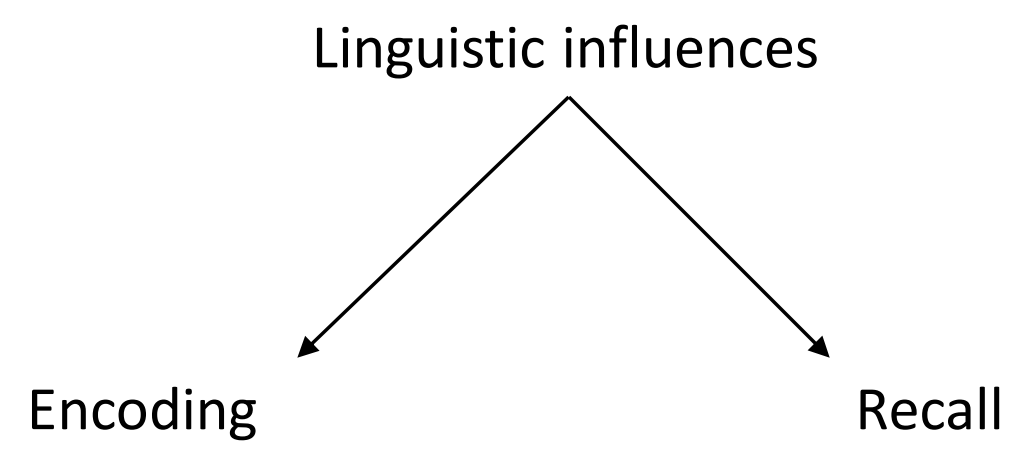

Language-based models of WM

Reconstruction models of WM

Martin, Saffran \& Dell (1996)

Majerus (2013, 2019) Hulme, Maughan \& Brown (1991) Schweickert (1993) 


\section{Introduction}

Language-based models: these influences should be observed already at the moment of encoding.

Reconstruction models: these influences should NOT be observed at this stage. 


\section{Procedure}




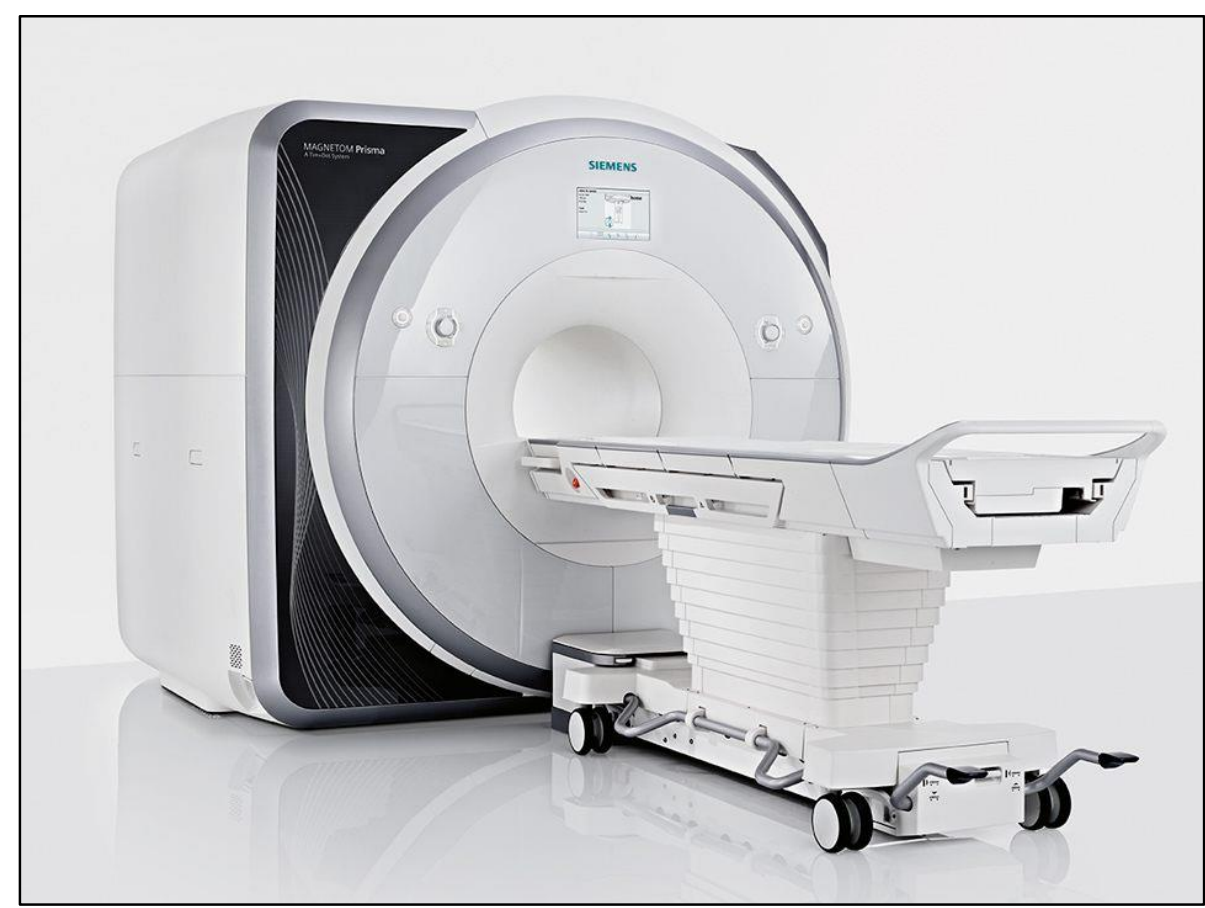

$N=29$

3 Tesla MRI scan

LIÈGE université
GIGA institute

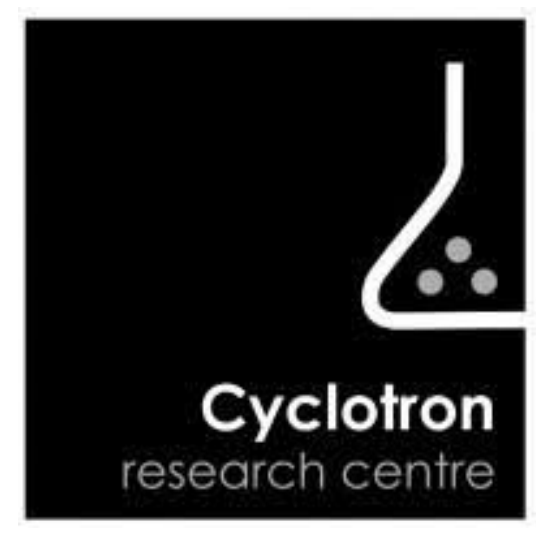




\section{Procedure}

Encoding conditions minimizing the opportunity to use reconstruction mechanisms.

$$
N=9 / 10 / 11 / 12
$$

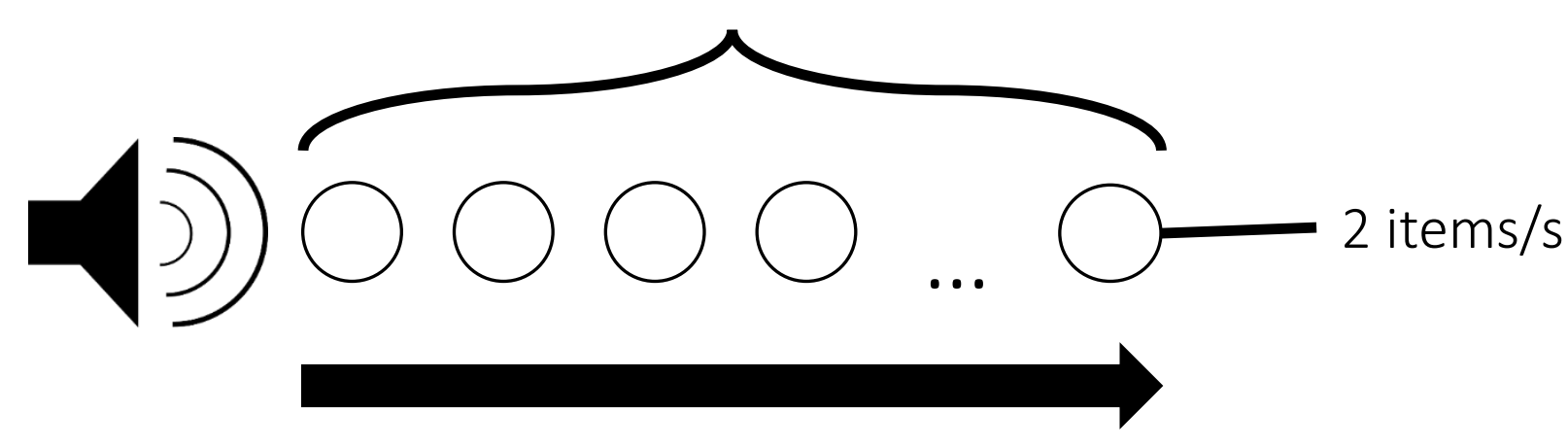




\section{Procedure}

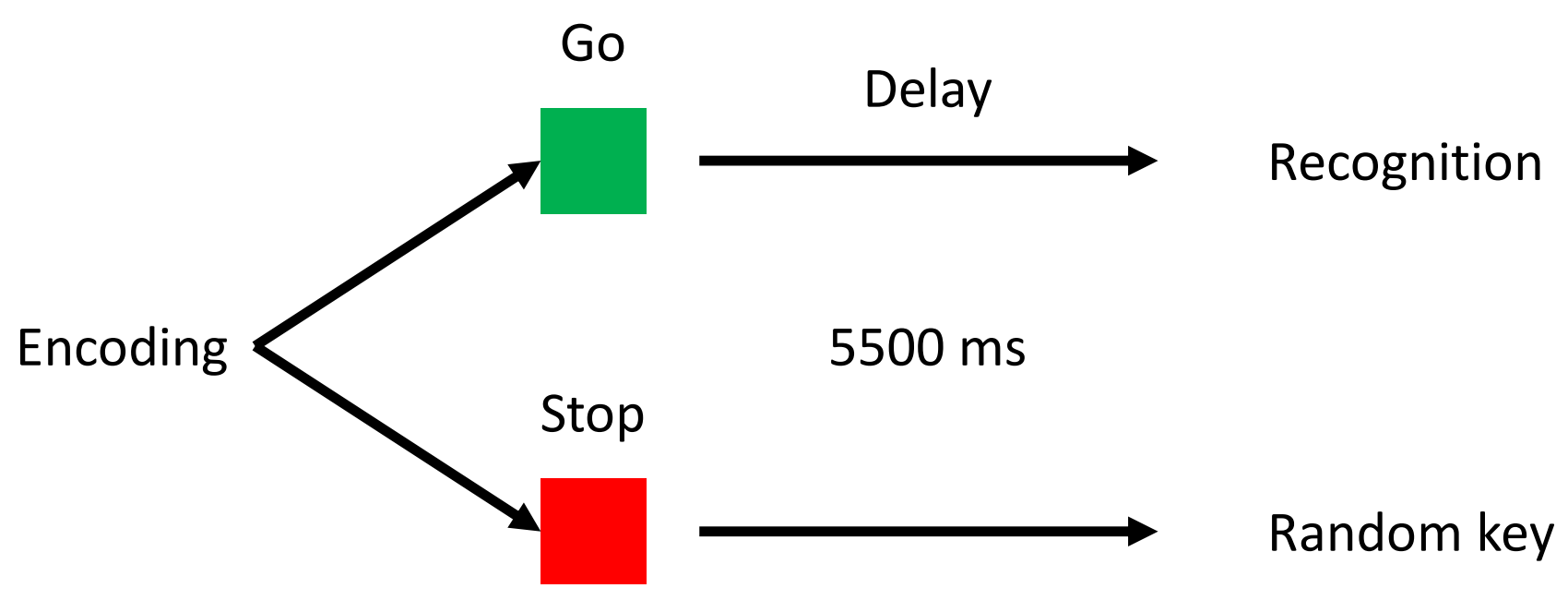


Leave-one-out cross-validation procedure

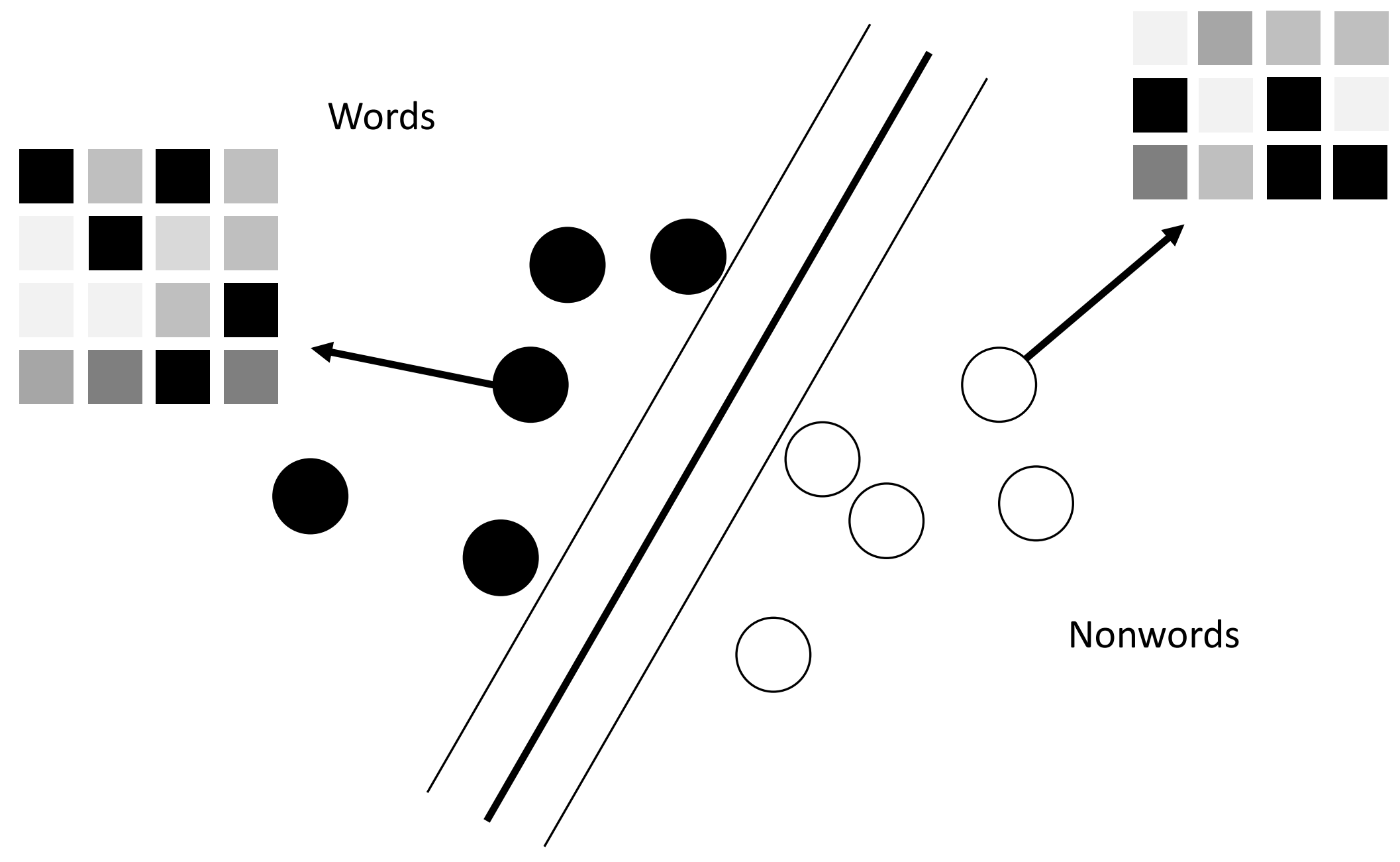


Results 
Semantic processing

Ventral language pathway

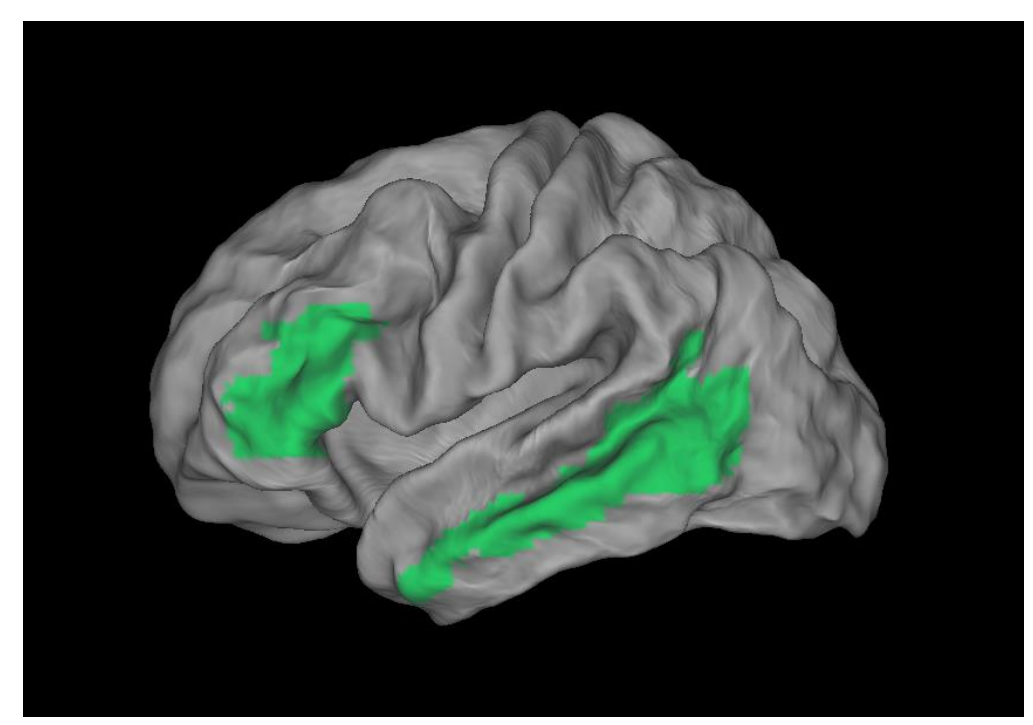

Phonological processing

Dorsal language pathway

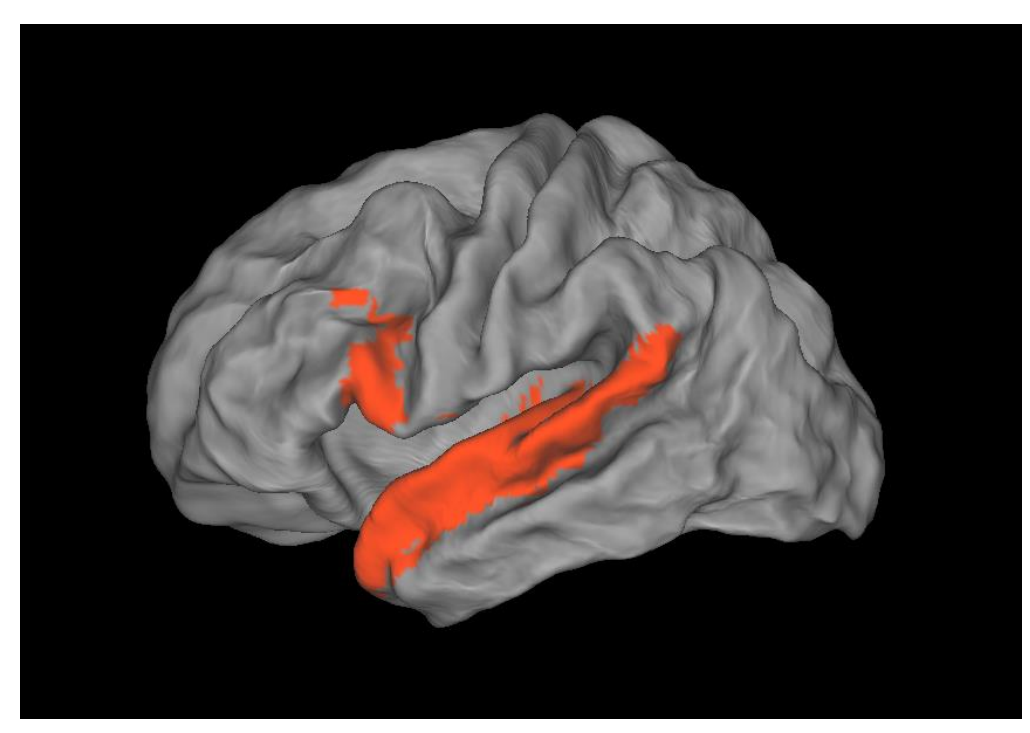

Friederici (2012)

Friederici \& Gierhan (2013)

Hickok \& Poeppel (2007) 
Semantic processing
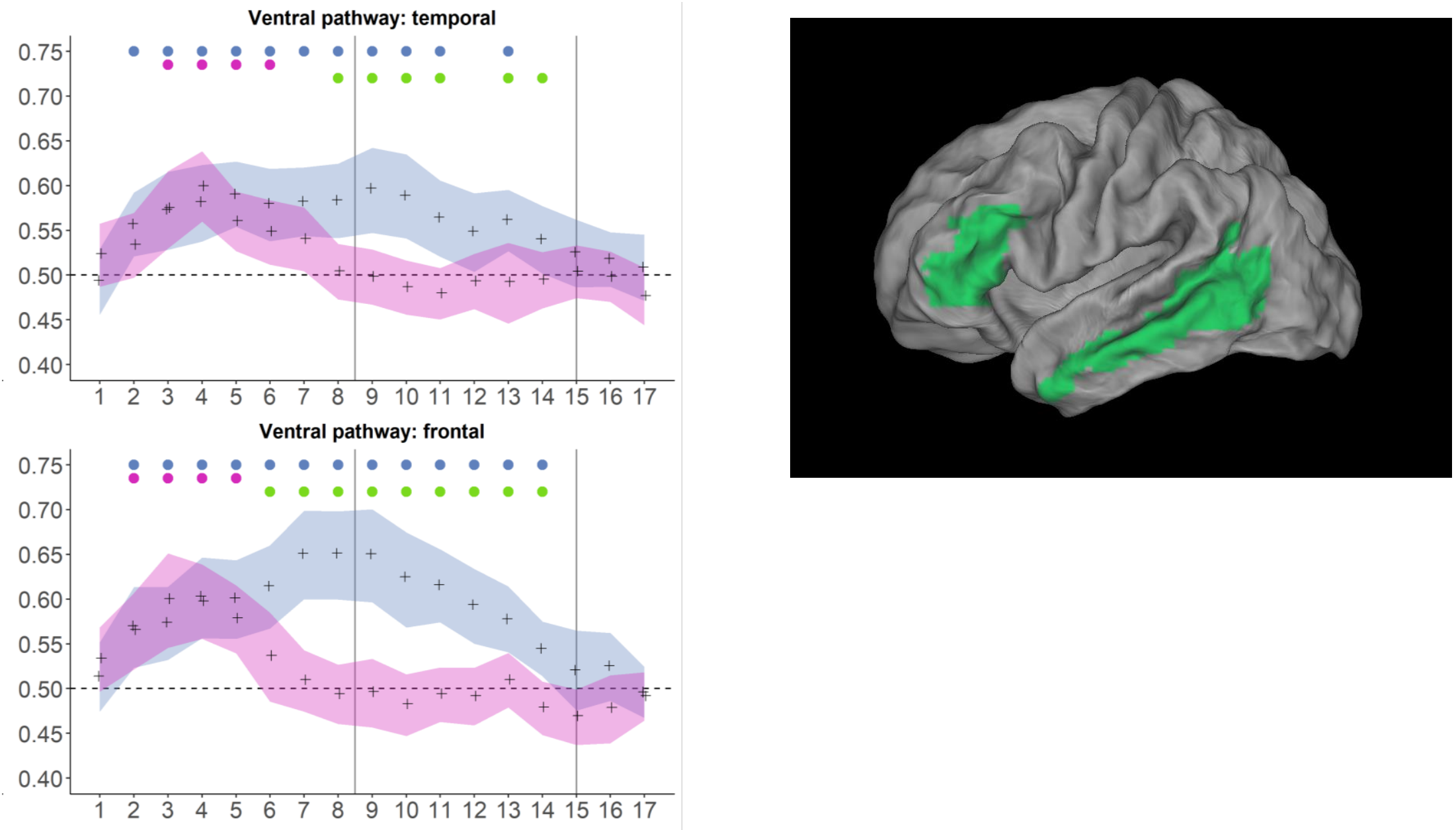

Note. The ribbons correspond to 95\% credible intervals of the mean. 
Semantic processing
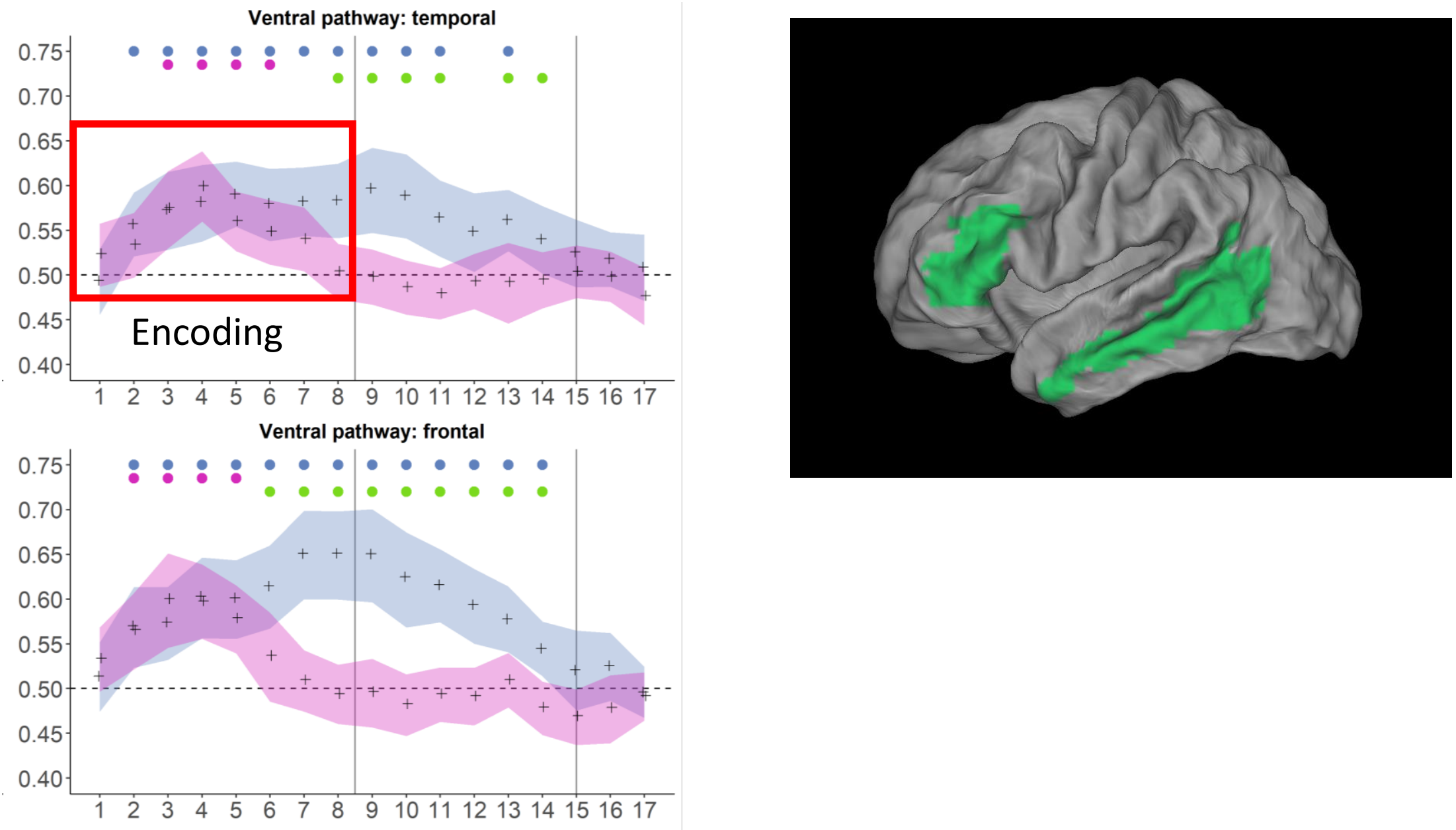

Note. The ribbons correspond to 95\% credible intervals of the mean. 
Semantic processing
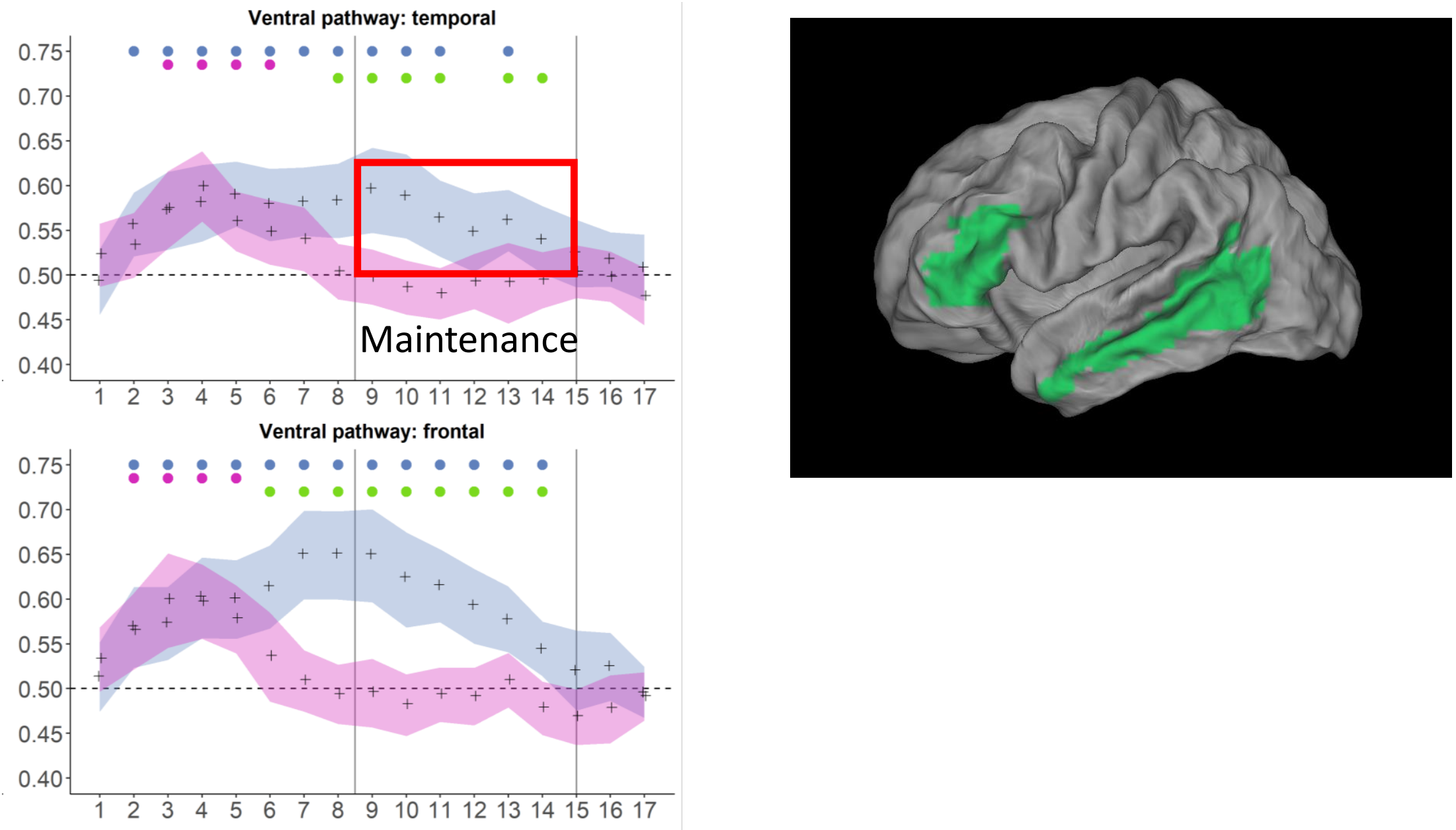

Note. The ribbons correspond to 95\% credible intervals of the mean. 
Semantic processing
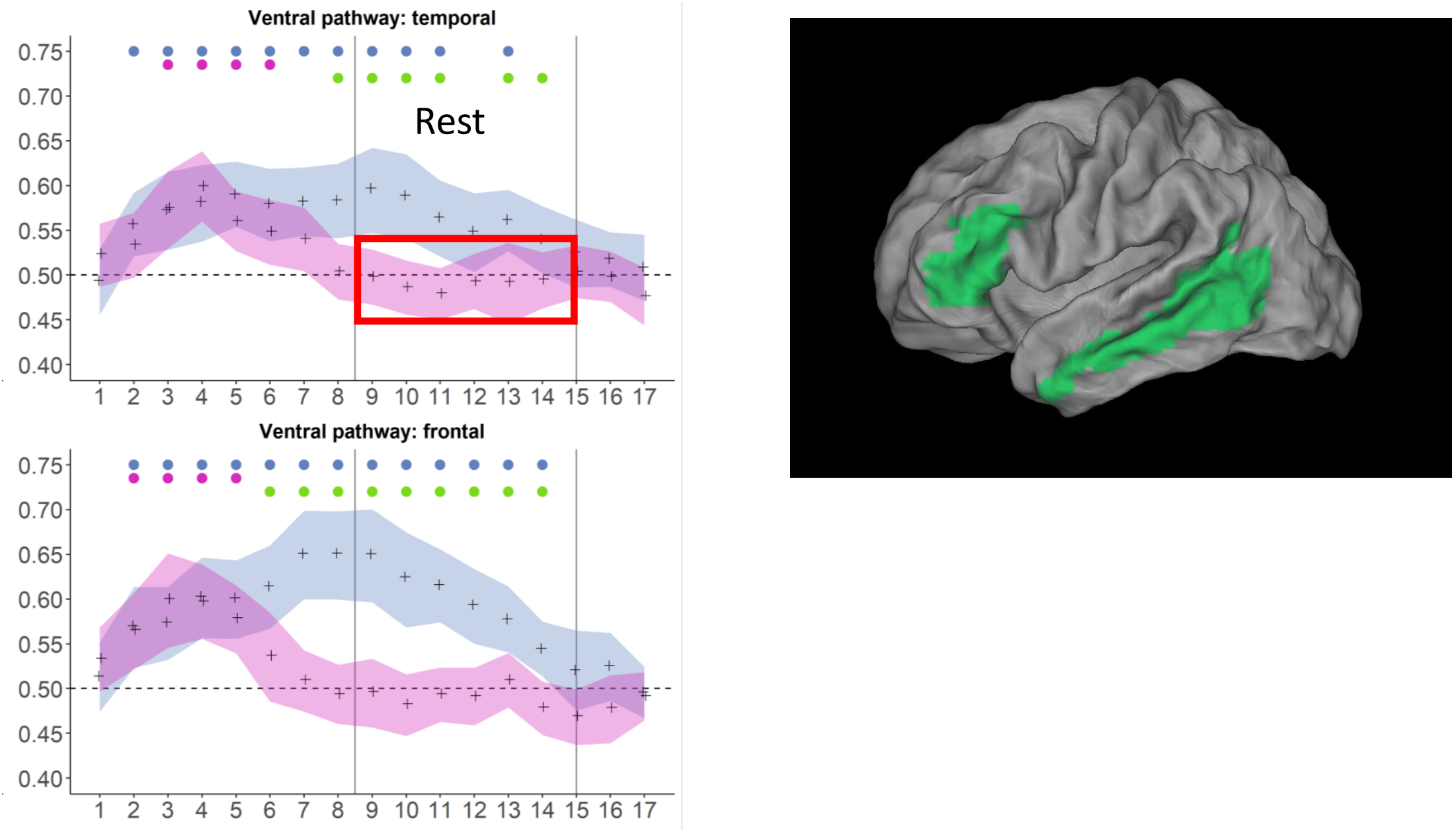

Note. The ribbons correspond to 95\% credible intervals of the mean. 


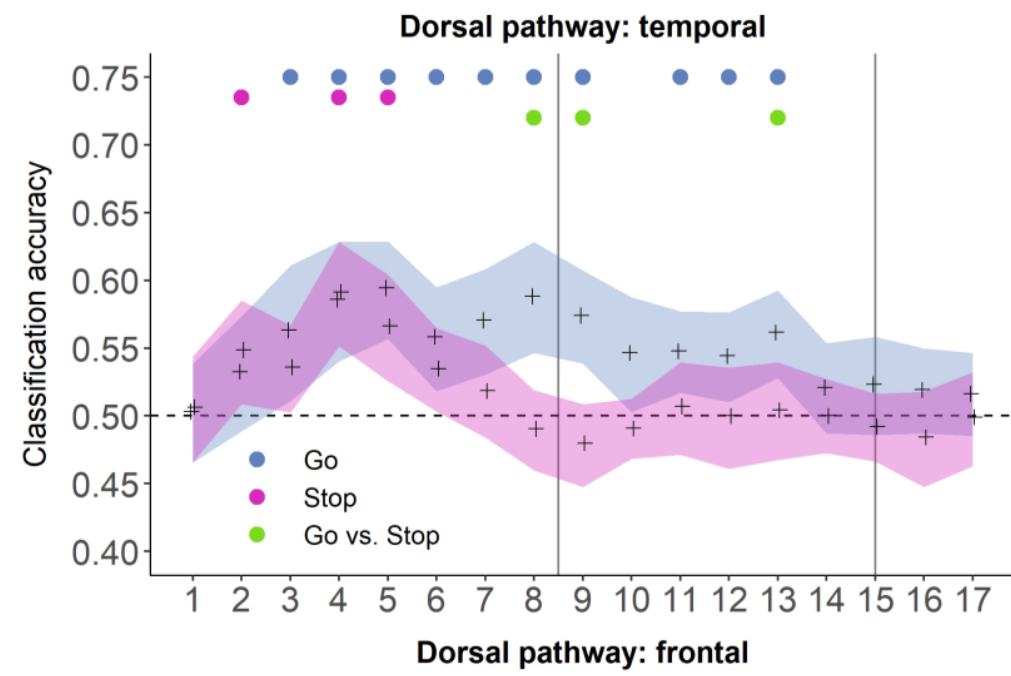

\section{Phonological processing}
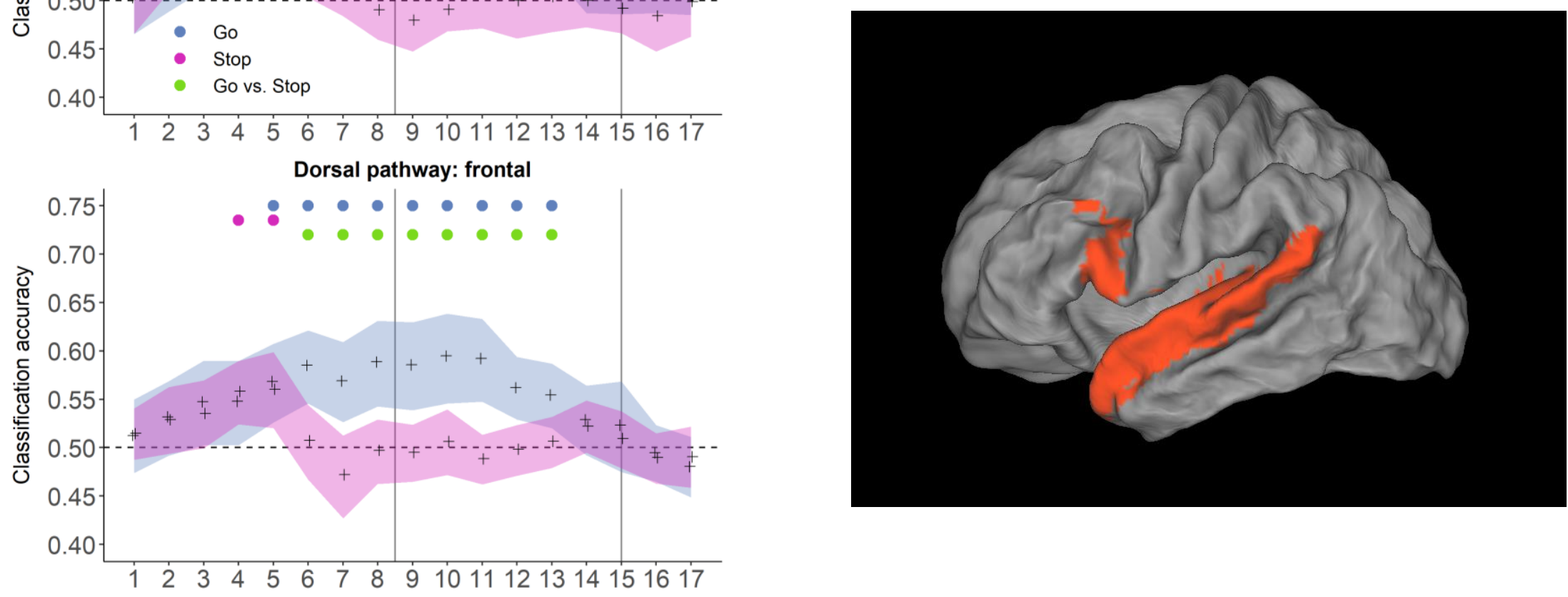

Note. The ribbons correspond to 95\% confidence intervals of the mean. 


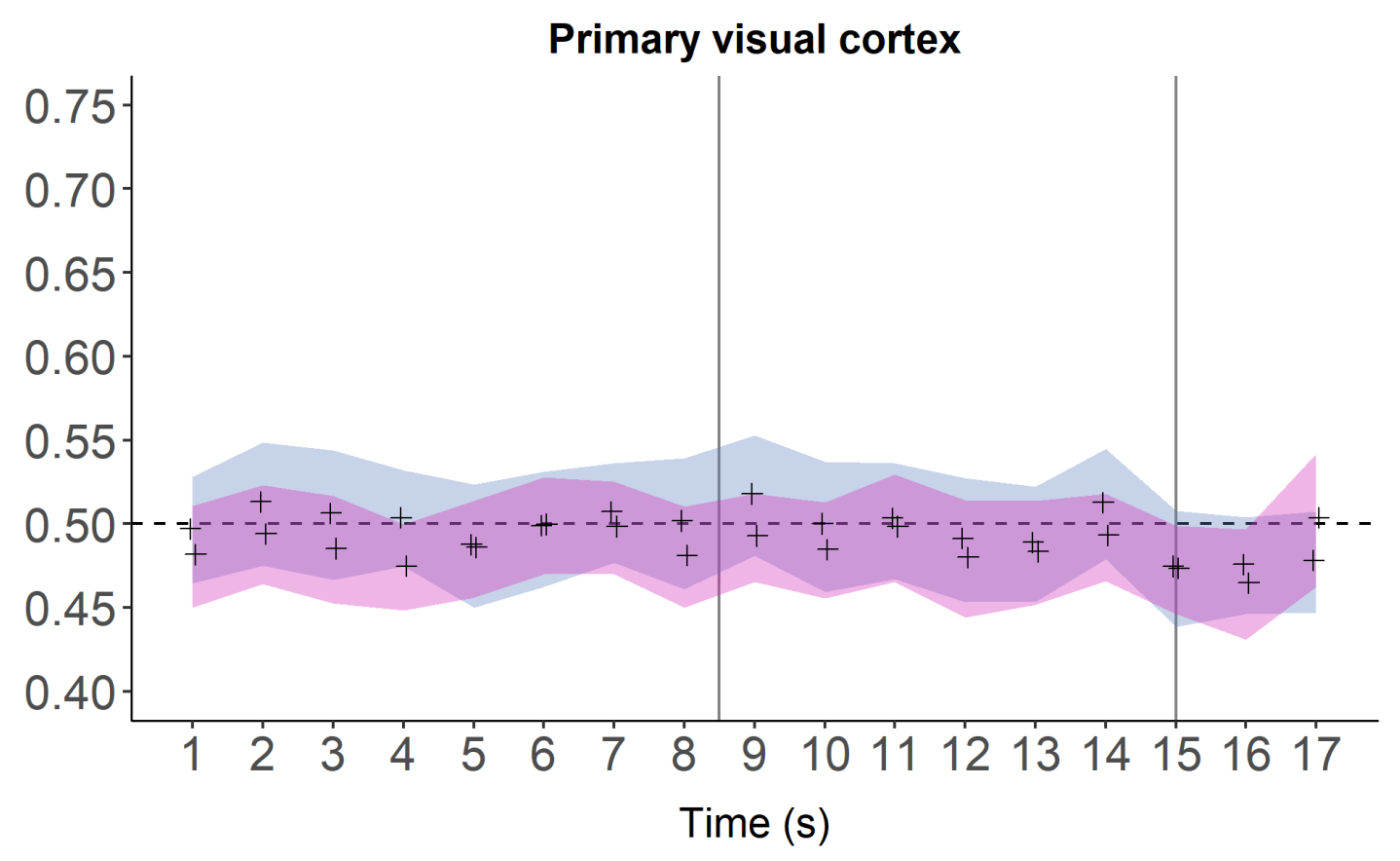




\section{Discussion}


Provide evidence for a direct involvement of linguistic cortices to access linguistic knowledge during WM processing.

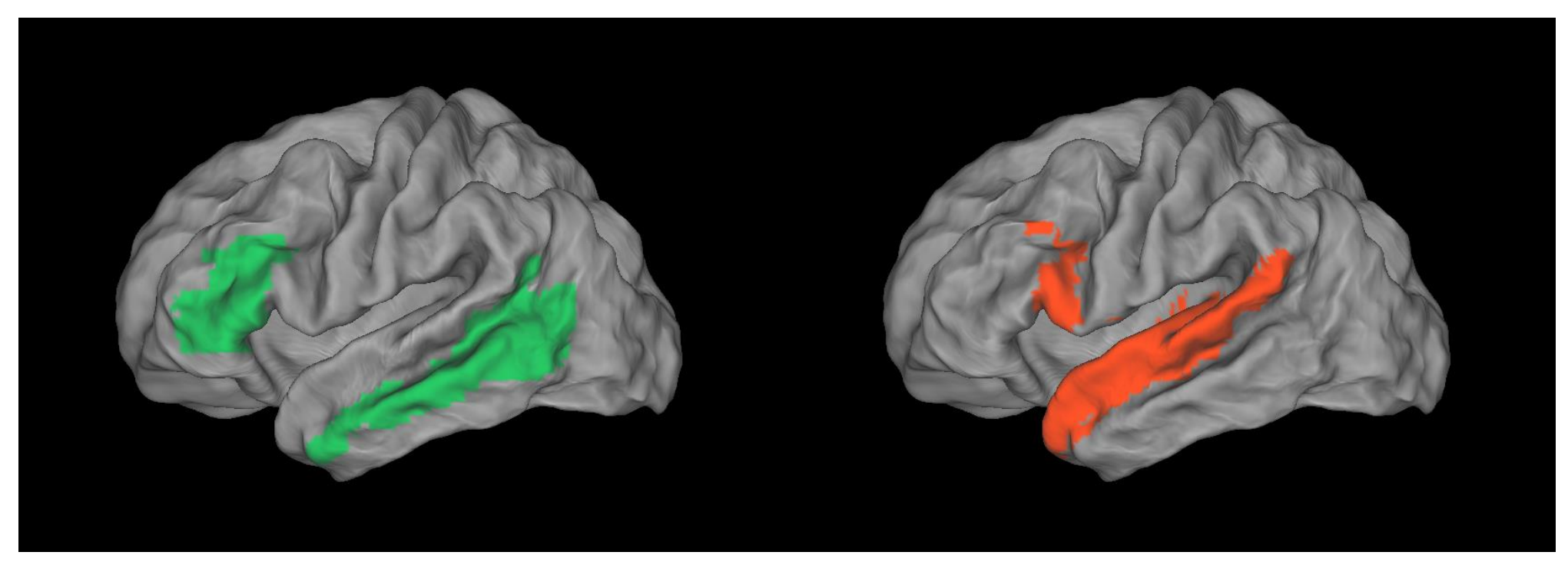




\section{Discussion}

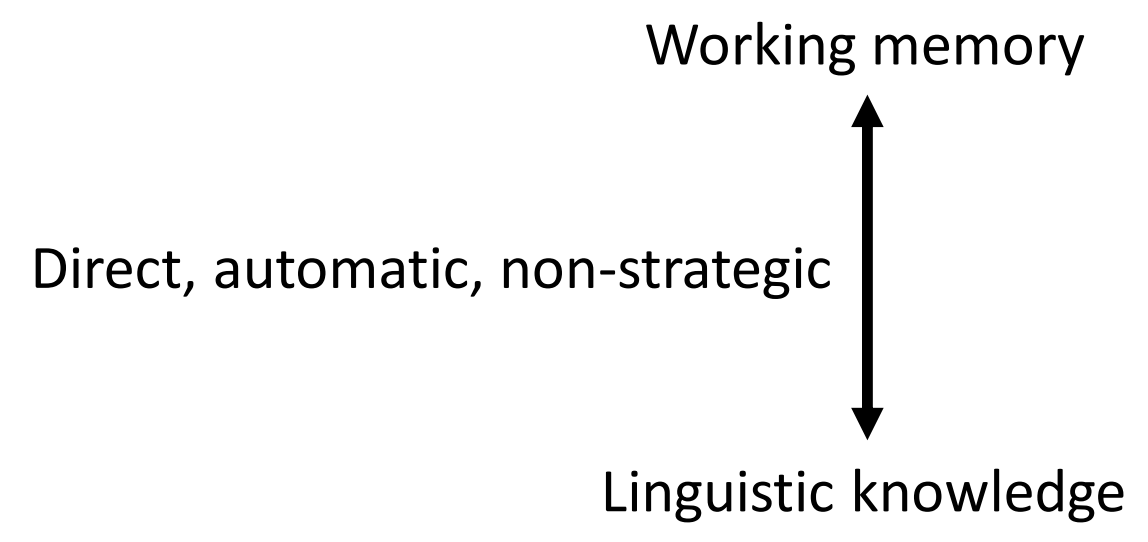




\section{Discussion}

These properties are also reflected in language processing.

The linguistic system is able to access linguistic knowledge very efficiently and rapidly, approximately around $\sim 50 \mathrm{~ms}$ (MacGregor et al., 2012). 


\section{Discussion}

Working memory

Cowan $(1995,2001)$

Martin, Saffran \& Dell (1996)

Majerus $(2013,2019)$

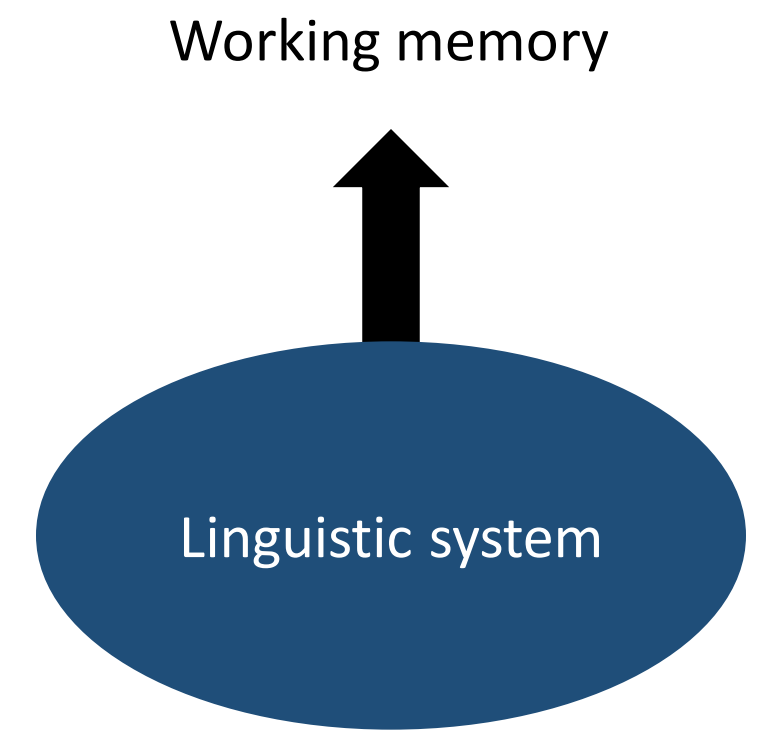




\section{Conclusion}

Theoretical models of verbal working memory need to consider the linguistic system as a core component. 
Thank you your attention 